\title{
SAVE PALMYRA. \\ LA DISTRUZIONE DEL PATRIMONIO ARCHEOLOGICO NEL VICINO ORIENTE. PERCHÉ E COME RICOSTRUIRE
}

\author{
Elena Pomar - Sapienza Università di Roma
}

The Museum of the Near East, Egypt and Mediterranean launched the "Save Palmyra" project for the awareness and knowledge of the damage that occurred in Syrian and Iraqi sites, in the last years. One of the aims of the project was to highlight the importance of $3 D$ technologies applied to archaeology.

Keywords: archaeology; cultural heritage; reconstruction; 3D technologies; safeguard

I tristi eventi che negli ultimi anni stanno tormentando alcuni dei paesi che custodiscono le vestigia di civiltà pre-classiche e i resti di città d'epoca romana e di monumenti islamici, hanno indotto il Museo del Vicino Oriente Egitto e Mediterraneo (MVOEM) ad affrontare il delicato e attuale tema della distruzione del patrimonio archeologico in Siria e in Iraq, attraverso un'esposizione dal titolo “Save Palmyra”, inaugurata con una tavola rotonda il 14 Maggio 2016.

\section{IL PROGETTO SAVE PALMYRA}

Il sito di Palmira, divenuto tristemente simbolo dello scempio che diversi luoghi di interesse storico e archeologico stanno subendo, è stato scelto come caso di studio specifico per la sua rilevanza storico-artistica e culturale. La mostra tematica, pur osservando più nel dettaglio i monumenti di Palmira, ha mostrato una panoramica generale dei danni arrecati anche ad altri importanti siti archeologici siriani e iracheni, tra i quali Dura Europos, Tell Mardikh/Ebla, Damasco e Nimrud/Kalkhu ${ }^{1}$.

Il progetto "Save Palmyra" è stato articolato in tre momenti: 1, il reperimento, la selezione e l'elaborazione del materiale grafico e bibliografico per l'allestimento del percorso espositivo, con il principale obiettivo di illustrare e valutare lo stato di conservazione e i danni subiti dai maggiori monumenti dall'inizio della guerra civile in Siria (2011) a oggi (§ 1.1.); 2, la realizzazione di un video 3D del Tempio di Baalshamin a Palmira e di quattro render (immagini ricostruite) dello stesso tempio, al fine di offrire la ricostruzione virtuale di uno dei più bei santuari palmireni oggi completamente distrutto ( $\S$ 1.2.); 3, l'organizzazione di una tavola rotonda inaugurale, allo scopo di partecipare all'ampio dibattitto circa la ricostruzione e il restauro dei monumenti antichi (§ 1.3.).

\subsection{L'esposizione di Save Palmyra al MVOEM}

Il percorso espositivo è stato articolato in modo da consentire al visitatore di approcciare il tema della mostra attraverso diversi registri informativi: la lettura di pannelli esplicativi (fig. 1); la visione di immagini selezionate del sito di Palmira e di alcuni dei suoi edifici ricostruiti con tecnologie $3 \mathrm{D}$ da diversi enti (§ 1.2.), presentate in scorrimento su

1 Danti 2015

ISSN 0393-0300

e-ISSN 2532-5159 
uno schermo; l'interazione con il materiale digitale ${ }^{2}$ su un touch screen, con la possibilità di avviare il video ricostruttivo del Tempio di Baalshamin e il tour virtuale tra le rovine di Palmira realizzato da Kaeamena $360^{\circ} 3$.

Sono stati realizzati, a cura di chi scrive, due pannelli e due roll up espositivi (bilingui italiano-inglese) che trattano, da un lato, la storia e la cultura siriana (e palmirena in particolare) in età romana, e, dall'altro, analizzano le principali problematiche relative alle distruzioni e ai danneggiamenti intenzionali subiti da monumenti, manufatti e interi siti archeologici ad opera dello Stato Islamico e in conseguenza degli scavi clandestini, esponenzialmente aumentati negli ultimi tempi ${ }^{4}$.

Nel primo roll-up, dal titolo "Palmyra, oasi nel deserto", vengono brevemente ripercorse le tappe storiche che hanno portato allo splendore della città nel II e III sec. d.C. e alla nascita del regno di Zenobia e del figlio Vallabato (267-272 d.C.); sono illustrati alcuni dei più grandiosi monumenti e il variegato pantheon palmireno, frutto dell'incontro e del sincretismo di diversi gruppi etnici e dei contatti con le prospere regioni circostanti.

Nel secondo pannello, “La Siria romana e l'impero palmireno", si trovano sintetizzati i processi storici che, partendo dall'età ellenistica, hanno condotto alla creazione della Provincia Romana di Siria nel 64 a.C. (successivamente interessata da diverse riforme imperiali che ne hanno modificato l'amministrazione e il nome) e gli eventi più salienti che hanno interessato la nuova provincia, fino al III sec. d.C.

Il terzo roll-up, "Save Palmyra! Un futuro per la memoria del passato”, è stato dedicato ai monumenti palmireni che più gravemente sono stati segnati dall'occupazione militare dei jihadisti del sedicente Stato Islamico. Tramite immagini satellitari, diffuse sul web soprattutto da organizzazioni come ASOR CHI (American Schools of Oriental Research. Cultural Heritage Initiatives $)^{5}$, e fotografie scattate all'indomani della liberazione della città ad opera delle truppe governative siriane e degli alleati russi (27 Marzo 2016), viene confrontato lo stato di conservazione dei monumenti e delle opere ospitate nel Museo Nazionale di Palmira prima e dopo i danneggiamenti e le distruzioni (fig. 2).

Nella seconda parte dello stesso pannello sono descritte alcune delle moderne tecnologie 3D (come stampanti 3D che riproducono in scala reale o ridotta oggetti e monumenti archeologici; programmi di modellazione con cui è possibile realizzare video e render ricostruttivi) che possono essere utilizzate come strumenti di salvaguardia della memoria di edifici e manufatti andati distrutti.

Nel quarto ed ultimo pannello sono state ripercorse le tappe dell'avvento dell'Isis nel governatorato di Homs e i danni arrecati ai siti siriani dichiarati patrimonio dell'UNESCO, come ad esempio Aleppo e Damasco, ma anche ad altri siti di fondamentale importanza come Ebla e Dura Europos, oggetto di volta in volta, non solo di distruzioni sistematiche

2 Il materiale digitale è anche accessibile dal sito del MVOEM www.lasapienzatojericho.it/Museo/PalmyraVideo.

3 www.kaemena360.com/360/PalmyraTour.

4 Si tratta della più grave crisi del patrimonio culturale dopo quella della Seconda Guerra Mondiale (Danti 2015, 135).

5 ASOR CHI (www.asor-syrianheritage.org) si occupa di monitorare siti archeologici e monumenti siriani tramite foto satellitari di Digital Glob e Skybox che vengono georeferenziate attraverso il software open source ARCHES (archesproject.org). 
ma anche di scavi clandestini e saccheggi ${ }^{6}$. Questi ultimi, sempre più numerosi, contribuiscono all'aumento del commercio illegale di antichità siriane (e irachene) sul mercato nero ${ }^{7}$.

\subsection{Il materiale interattivo e le tecnologie 3D applicate all'archeologia}

Al fine di aprire lo spazio museale a nuove forme di comunicazione in grado di richiamare l'attenzione di un pubblico più ampio si è scelto, in occasione dell'evento "Save Palmyra", di produrre materiale digitale interattivo e controllato dal punto di vista scientifico.

Sono stati realizzati, a cura di chi scrive, un video 3D e quattro immagini render del Tempio di Baalshamin a Palmira. Questo monumento è stato scelto come caso-studio data la sua importanza e rappresentatività, nonché per la volontà di conservarne e divulgarne la memoria, straordinariamente ben custodita dal tempo prima dell'esplosione avvenuta per mano dei jihadisti nell'Agosto 2015.

La modellazione 3D del tempio è stata fatta sulla base delle piante e dei prospetti pubblicati da P. Collart nella relazione di scavo ${ }^{8}$, di cui sono state adottate le proposte ricostruttive per quanto riguarda la sommità e la copertura. Il modello tridimensionale ha restituito un'immagine dell'edificio come doveva apparire al momento della sua costruzione, nel II sec. d.C.

In una prima fase sono stati vettorializzati le piante e i prospetti su AutoCad, successivamente questi sono stati esportati su un programma di modellazione 3D (SketchUp). Il modello tridimensionale è stato costruito estrapolando tutti i dati relativi alle dimensioni del monumento e di tutte le sue parti (crepidoma, colonne, pilastri, fregio, architrave ecc.) dai rilievi. Una volta creato il modello, parte dell'elaborazione 3D è stata dedicata alla resa grafica dei materiali costruttivi e della loro messa in opera. In fase di renderizzazione, al fine di ottenere un effetto più realistico, è stata impostata una sorgente di luce, così da caratterizzare meglio le superfici e creare le ombre (fig. 3). Con lo stesso programma è stato realizzato un video che riproduce un percorso virtuale intorno al tempio e al suo interno, dove si trovava lo splendido thalamos. Infine sono state costruite tre immagini tramite l'inserimento fotografico del modello renderizzato (fig. 4) e una di dettaglio dell'interno del tempio (fig. 5), in modo da mostrare il monumento ricostruito nel suo contesto paesaggistico.

Lo studio di piante, sezioni, prospetti e fotografie, documentati nelle pubblicazioni di scavo, costituisce la risorsa primaria, con cui può essere realizzata la ricostruzione scientifica, digitale (tramite modellazione 3D) ${ }^{9}$ e/o fisica (con stampanti 3D), dei

6 Per una trattazione completa e approfondita si veda ad esempio Guidetti - Perini 2015

7 In merito al grave problema del traffico illecito di antichità si fa menzione ad alcuni programmi e sistemi di tutela del patrimonio archeologico mobile disponibili su internet, come le red lists redatte dal International Council Of Museums (ICOM) e il Palestine Archeological Database and Information System (PADIS), creato in cooperazione tra l’Università di Roma La Sapienza e la Direzione Generale delle Antichità Palestinesi (http://www.lasapienzatojericho.it/padis/).

8 Collart- Vicari 1969.

9 Un esempio notevole di ricostruzione 3D di un monumento archeologico è costituito dal video presentato dal Metropolitan Museum, che simula un percorso all'interno del Palazzo Nord-Ovest di Nimrud, costruito da 
monumenti. Il 3D rappresenta una delle molteplici tecnologie moderne che possono essere impiegate per lo sviluppo di nuovi metodi e sistemi di tutela e salvaguardia della memoria del patrimonio culturale a rischio, che se non fisicamente potrà essere fruibile in forma digitale ${ }^{10}$.

Nell'ambito della ricostruzione virtuale, per quanto riguarda Palmira è stata creata, ad opera dell'organizzazione "New Palmyra" ${ }^{11}$, una piattaforma online per la raccolta di dati e materiali (sia digitali che fotografici), mentre è stato avviato un programma di ricostruzione fisica in scala, tramite l'utilizzo di sofisticate stampanti 3D, di alcuni monumenti, il primo dei quali a essere stato riprodotto in marmo dall’Institute of Digital Archeology di Oxford è l’Arco di Trionfo di Settimio Severo, collocato a Trafalgar Square il 19 Aprile 2016.

\subsection{La tavola rotonda inaugurale: il dibattito sulla ricostruzione}

L'esposizione è stata inaugurata il giorno 14 Maggio 2016 con un evento dedicato al quale sono intervenuti il presidente, Prof. Marina Righetti, e il direttore, Prof. Giorgio Manzi, del Polo Museale della Sapienza, il direttore del Museo dell'Arte Classica, Prof. Marcello Barbanera, il Prof. Paolo Matthiae, la giornalista Cinzia Dal Maso e il direttore del Museo del Vicino Oriente, Egitto e Mediterraneo, Prof. Lorenzo Nigro.

L'evento è stato organizzato in due sezioni: la prima apertura al pubblico e la tavola rotonda. Quest'ultima è stata dedicata alla difficile questione della ricostruzione in situ di monumenti distrutti (fig. 6), affrontata in un dibattito aperto tra oratori e pubblico ${ }^{12}$.

L'evento si inserisce nel più ampio programma volto all'accrescimento della consapevolezza e alla sensibilizzazione dell'opinione pubblica sulla necessità di preservare le testimonianze materiali di antiche culture e di limitare i danni del patrimonio archeologico immobile (siti e monumenti) e mobile (manufatti e reperti) ${ }^{13}$.

La questione della ricostruzione materiale di edifici distrutti, che deve tener conto della sovranità del paese interessato, del coordinamento dell’UNESCO e della collaborazione della comunità scientifica internazionale, è particolarmente delicata e complessa e va oltre limiti del presente contributo ${ }^{14}$.

Assurnasirpal II nel IX sec. a.C. (www.metmuseum.org/metmedia/video/collections/ancient-near-easternart/northwest-palace-nimrud).

10 L'emergenza attuale in merito alla tutela del patrimonio archeologico dei paesi in stato di guerra o di crisi, ha condotto la comunità scientifica internazionale a proporre nuovi sistemi di documentazione, divulgazione e ricostruzione, sfruttando le potenzialità offerte dalle moderne tecnologie (si veda Jamieson 2016 e Perini Cunliffe 2015).

11 www.newpalmyra.org.

12 È stato pubblicato un articolo dalla rivista Archeostorie, sull'inaugurazione dell'esposizione e sulla tavola rotonda tenuta il 14 Maggio, disponibile online (www.archeostorie.it/la-versione-di-archeostorie/salvarepalmira).

13 Per una trattazione completa riguardante le distruzioni del patrimonio culturale si veda il libro recentemente pubblicato da P. Matthiae (2015).

14 Sul tema della ricostruzione è stata inaugurata, il 7 Ottobre 2016 presso il Colosseo, la mostra "Rinascere dalle distruzioni. Ebla, Nimrud, Palmira”, dove saranno esposte fino all'11 Dicembre, le riproduzioni in scala reale della Sala del Grande Archivio del Palazzo di Ebla, il soffitto del Tempio di Bel a Palmira e il colossale toro androcefalo del Palazzo Nord-Ovest di Nimrud, tutti gravemente danneggiati o distrutti dalla furia dell'Isis. 


\section{CONSIDERAZIONI FINALI}

L'esposizione 'Save Palmyra' ha contribuito ad evidenziare ulteriormente il potenziale delle tecnologie 3D applicate all'archeologia, che consentono, quando sotto il diretto controllo di esperti, quali archeologi, architetti e storici dell'arte, di restituire informazioni e di ricostruire i monumenti e i reperti perduti sulla base di dati scientifici, con la garanzia di un errore minimo.

Le moderne tecnologie 3D offrono la possibilità di una ricostruzione virtuale o materiale di oggetti e monumenti, che soddisfi il bisogno di fruizione della conoscenza e metta a disposizione di chiunque la possibilità di osservare e studiare opere in luoghi lontani o non più esistenti, com'è il tragico caso dei templi di Bel e Baalshamin e di alcune tombe monumentali a Palmira. Inoltre, i prodotti digitali hanno il vantaggio, da un lato, di essere facilmente accessibili, costituendo un efficace mezzo di comunicazione sia per la comunità scientifica che per il vasto pubblico, e, dall'altro, di essere facilmente immagazzinati e conservati.

L'inaugurazione dell'esposizione ha fornito un elemento di riflessione sulla dolente questione della distruzione sistematica del patrimonio culturale, di cui si è potuto evidenziare un secondo aspetto, emergente soprattutto nei paesi in stato di guerra: il contrabbando di manufatti antichi ${ }^{15}$ e le implicazioni che ne derivano. Questo, infatti, comporta alcune conseguenze drammatiche, da un lato il ricavato delle vendite illecite costituisce uno dei maggiori proventi dell'Isis e degli altri gruppi coinvolti nel conflitto ${ }^{16}$, dall'altro la rottura della connessione tra reperto e contesto di rinvenimento compromette nuove conoscenze e comporta la perdita di un patrimonio archeologico.

Di fronte a uno stato di crisi di tali proporzioni scaturisce la necessità di affrontare il tema sulla ricostruzione materiale dei monumenti distrutti, che è stato oggetto del dibattito cui sono stati invitati a partecipare ospiti illustri, specialisti e archeologi. L'importanza e al tempo stesso la delicatezza dell'argomento possono indurre alla coesistenza di punti di vista che difficilmente riescono a conciliarsi dal punto di vista scientifico, ma accomunati dalla volontà di preservare la memoria: da un lato si incontra il desiderio di donare nuovo splendore ai siti privati dei loro monumenti e dare la possibilità al pubblico di goderne (come si vorrebbe fare proprio per Palmira); dall'altro vi è il bisogno di lasciare traccia, anche come forma di denuncia, di un assurdo momento storico che è parte come tutti gli altri del ciclo della storia e che come testimoni della sua esistenza non lascerà splendide costruzioni, ma crimini e sacrileghe distruzioni.

15 Il mercato nero di antichità è alimentato con oggetti e manufatti provenienti da furti nei musei e collezioni, e recuperati tramite pratiche di scavo illegali, il numero dei quali è in forte aumento a causa dell'impossibilità da parte delle autorità locali di proteggere i siti archeologici. Si veda su questo tema Mueller 2016.

16 Matthiae 2015. 


\section{BIBLIOGRAFIA}

COLLART, P. - ViCARI, J.

1969 Le sanctuaire de Baalshamin à Palmyre: Topographie et architecture. Vol II (Institut suisse de Rome), Roma 1969.

DANTI, M.D.

2015 Ground-Based Observations of Cultural Heritage Incidents in Syria and Iraq: Near Eastern Archaeology 78/3 (2015), pp. 132-141.

GuidetTi, M. - PERINI, S.

2015 Civil War and Cultural Heritage in Syria, 2011-2015. (Syrian Studies Association Bullettin 20/1, 2015).

JAMIESON, A. (ed).

2016 Collections at Risk: Sustainable Strategies for Managing Near Eastern Archaeological Collections. Proceedings of the 9th International Congress on the Archaeology of the Ancient Near East, Vol. I, Wiesbaden 2016, pp. 449-463.

MatThiae, P. (ed).

2015 Distruzioni saccheggi e rinascite. Gli attacchi al patrimonio artistico dall'antichità

MUELLER, T. all'Isis, Milano 2015.

2016 Il saccheggio della Storia: National Geographic 37/6 (2016), pp. 2-25.

PERINI, S. - CunLIFFE, E.

2015 Towards a protection of the Syrian cultural heritage: a summary of the national and international responses, vol. III (Sept 2014 - Sept 2015) (Heritage for Peace), Girona 2015.

\section{SITOGRAFIA}

$\begin{array}{lll}\text { Archeostorie } & \text { archeostorie.it } \\ \text { ARCHES } & \text { archesproject.org } \\ \text { ASOR CHI } & \text { asor-syrianheritage.org } & \\ \text { ICOM Red Lists } & \text { icom.museum/programmes/fighting- } & \text { illicit-traffic/red-list/ } \\ \text { Kaemena } 360^{\circ} & \text { kaemena360.com/home/360o-city- } & \text { tours/palmyra } \\ \text { Metropolitan Museum } & \text { metmuseum.org } & \\ \text { MVOEM } & \text { lasapienzatojericho.it/Museo } & \\ \text { New Palmyra } & \text { newpalmyra.org } \\ \text { PADIS } & \text { lasapienzatojericho.it/padis }\end{array}$




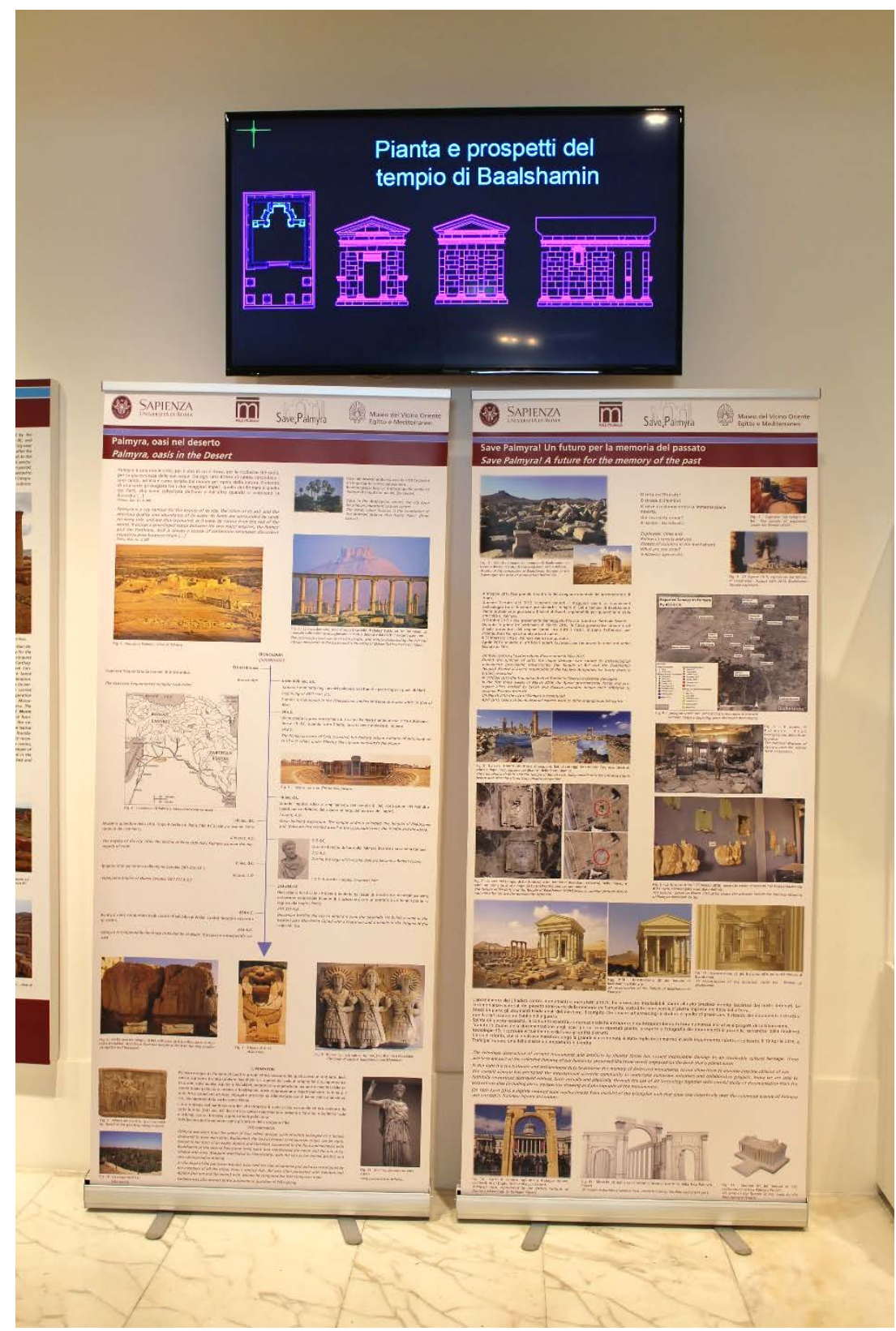

Fig. 1 - Inizio del percorso espositivo di Save Palmyra presso il MVOEM, con i pannelli dedicati a Palmira e lo schermo con le immagini di supporto selezionate, presentate in scorrimento. 


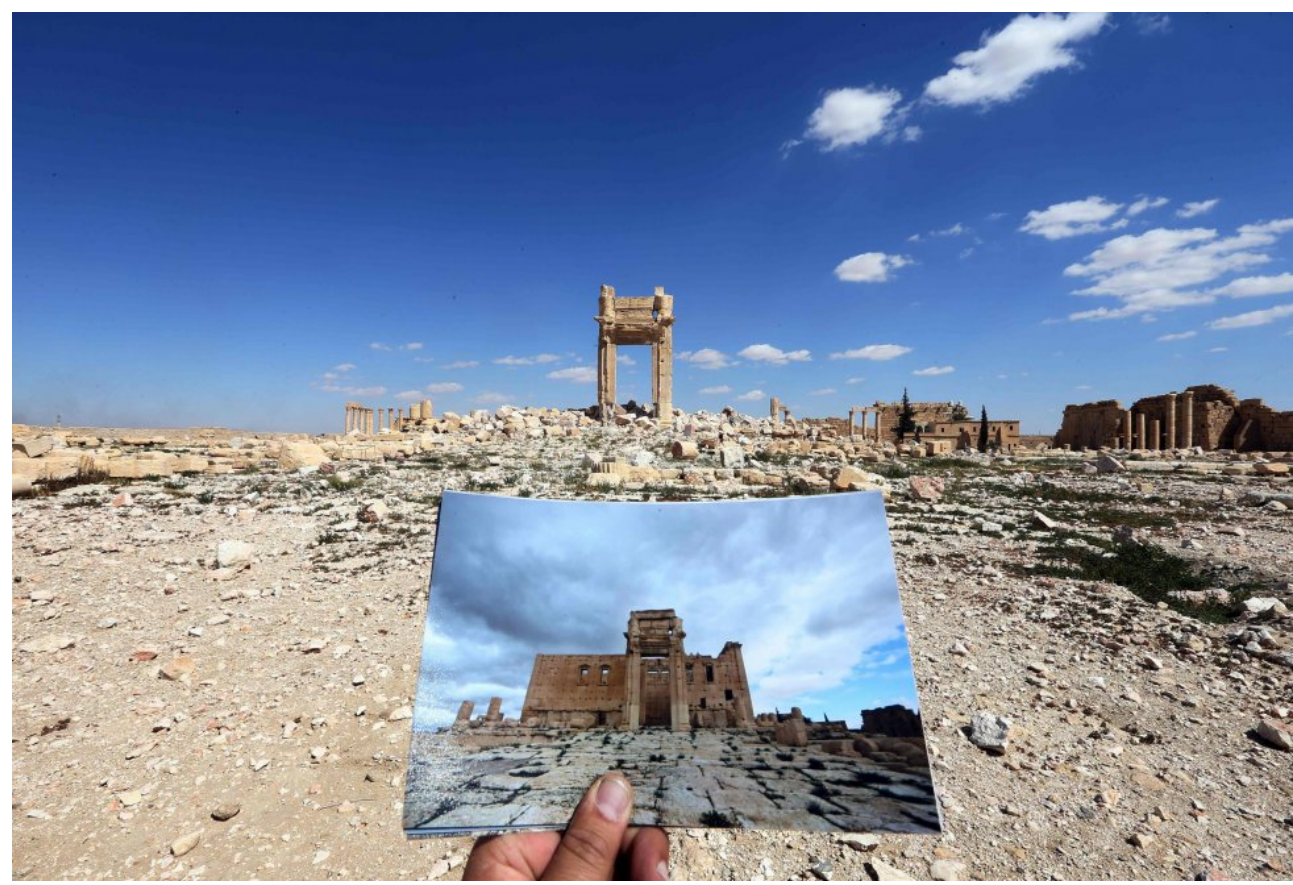

Fig. 2 - il tempio di Bel a Palmira confrontato prima e dopo l'occupazione dei jihadisti dell'IS (Getty images).

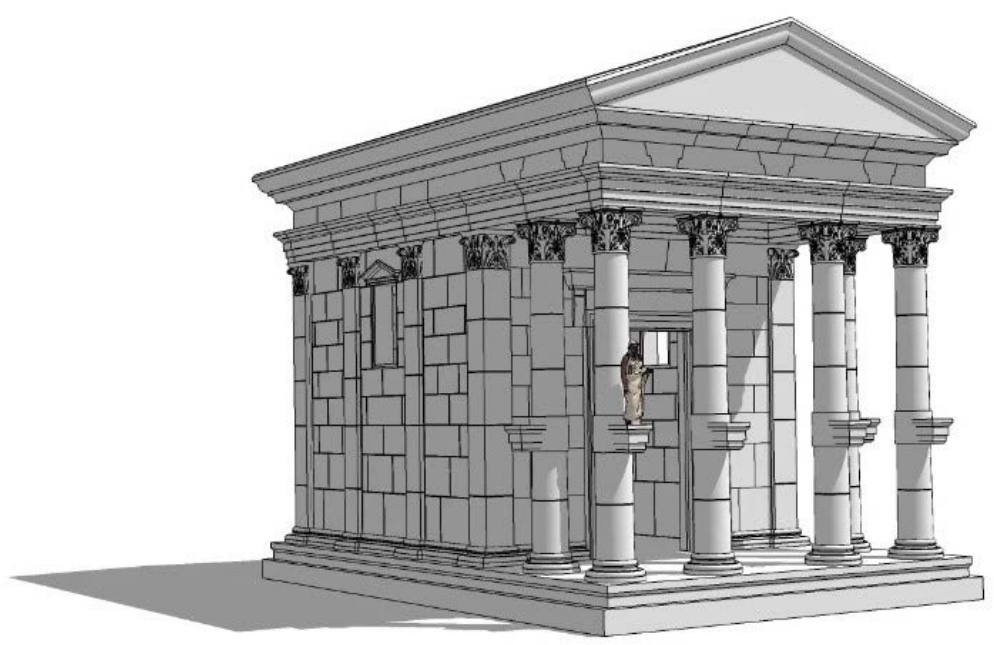

Fig. 3 - Modello ricostruttivo 3D del tempio di Baalshamin a Palmira. 


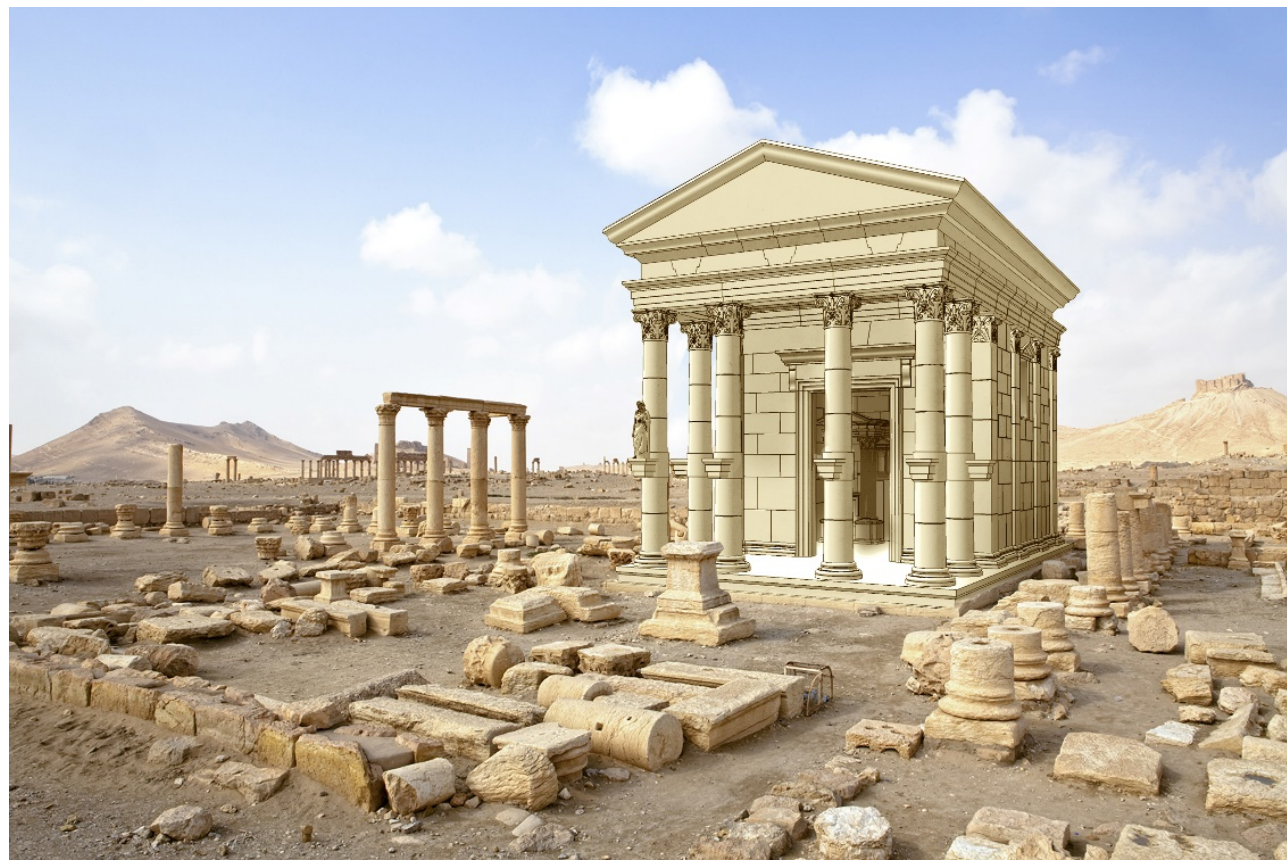

Fig. 4 - Render del Tempio di Baalshamin a Palmira, creato tramite inserimento fotografico.

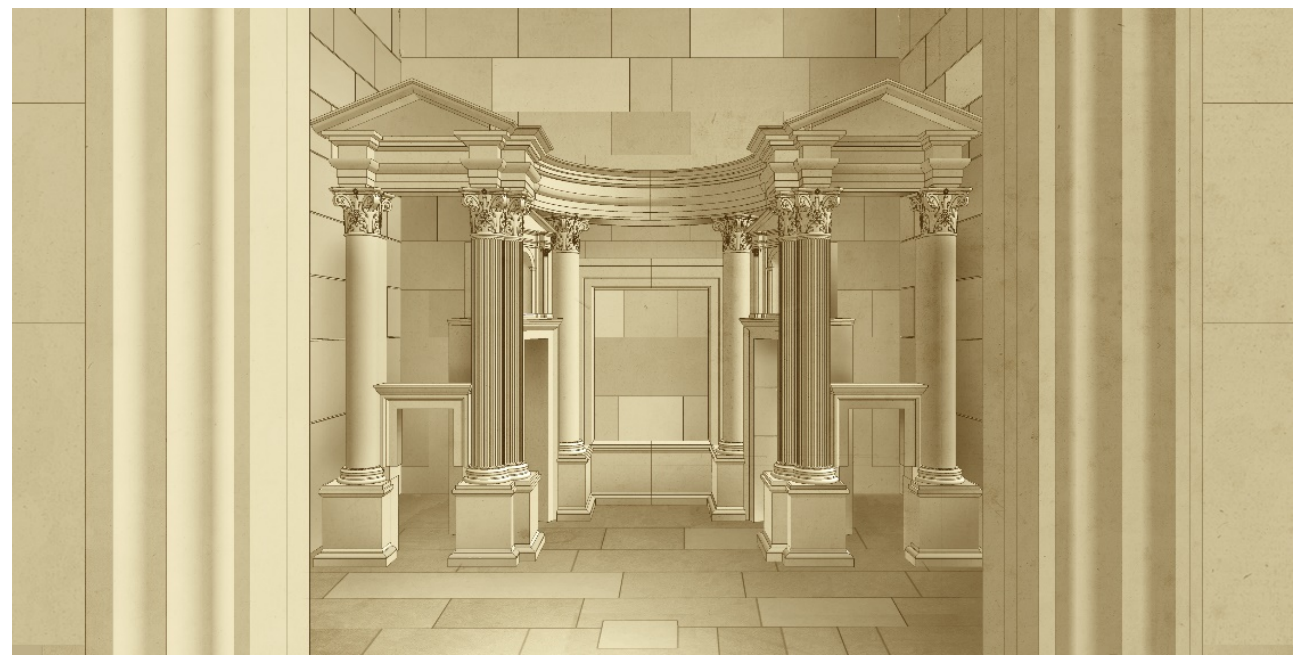

Fig. 5 - Render del thalamos che si trovava all'interno del Tempio di Baalshamin. 


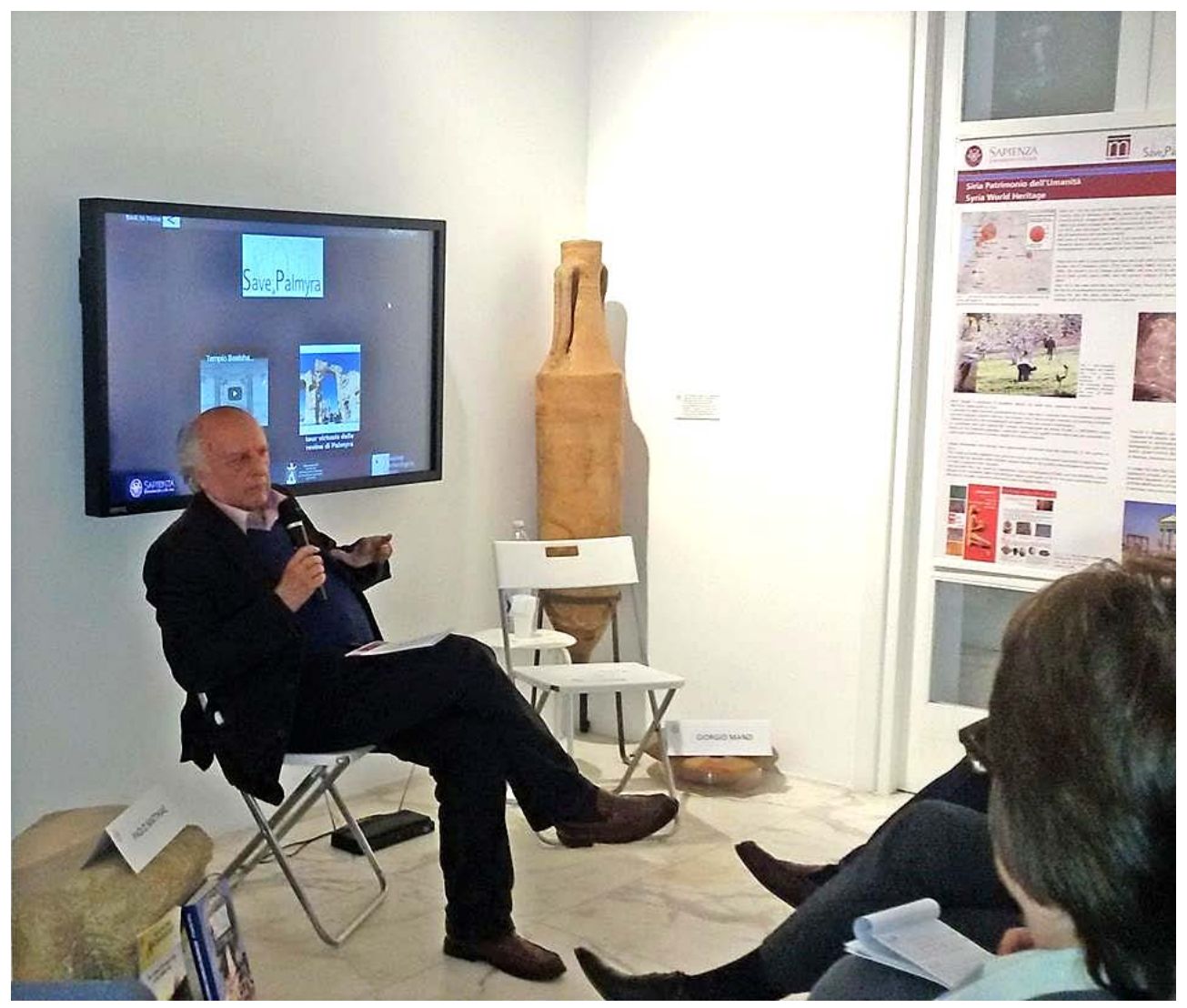

Fig. 6 - L'intervento del Prof. Paolo Matthiae alla tavola rotonda che ha avuto luogo il 14 Maggio 2016 presso il MVOEM. 